\title{
Random, Scattered and Asymmetric Distribution of Fixational Eye Movement - Experimental Evidence
}

\author{
Zhao Songnian ${ }^{1}$, Cheng Zhongbin ${ }^{2}$, Wang Fengjiao ${ }^{2}$, Zou Qi ${ }^{2, *}$ \\ ${ }^{1}$ LAPC, Institute of Atmospheric Physics, Chinese Academy of Sciences, Beijing 100029, China \\ ${ }^{2}$ School of Computer and Information Technology, Beijing Jiaotong University, Beijing 100044, China \\ *Corresponding author: qzou@bjtu.edu.cn
}

\begin{abstract}
How to determine the test chart is very important in fixational eye movement (or microsaccades) experiment, in this paper, the selected test image can provide important information about cognitive psychology and visual information processing, especially including a random dot stereogram as a test image to test the eye movement trajectory of the subjects. According to the computer vision and image feature analysis, we can predict and determine the obvious feature region in the test chart, then, compared with the eye movement trajectory of the subjects. There is a big difference between the eye movement trajectories of each participant, and the distribution of the fixation points is very random, scattered and asymmetric, which cannot be attributed to a certain statistical distribution and cannot determine their statistical parameters, and for this reason this paper suggests that the significant test for eye movement should be an interval estimation, and the specific interval estimates are given, and also points out that the microsaccade is equivalent to the conversion between the frames of visual images; the blinks of eyes are the conversion between the various primitives in visual images. These novel results are valuable for the study of cognitive psychology and vision information processing.
\end{abstract}

Keywords: fixation point, horizontal flip of image, eye movement trajectory, visual perception

Cite This Article: Zhao Songnian, Cheng Zhongbin, Wang Fengjiao, and Zou Qi, "Random, Scattered and Asymmetric Distribution of Fixational Eye Movement - Experimental Evidence." Research in Psychology and Behavioral Sciences, vol. 6, no. 1 (2018): 15-26. doi: 10.12691/rpbs-6-1-3.

\section{Introduction}

Human fixational eye movement (or microsaccades) research is closely related to the cognitive psychology, neuroscience and visual information processing. By using computational modeling, eye tracking, and brain imaging, it is able to understand how vision, attention, cognition, and motor control interact dynamically to produce the complex eye-movement behavior and it is also able to observe when humans read or perform other complex realworld tasks or looking for an object in a scene.

But, currently the study of human eye movement is still in its early stages, and the exact role of eye movements in cognitive neuroscience and cognitive psychology remains unclear. Psychologists and cognitive scientists' views differ on this issue. We designed four different eye movement experiments to investigate participants' eye movement trajectories when they viewed different images including symmetrical pictures, because the test images were representative and typical. Participants included European and American graduate students and Chinese students, allowing the experimental results to have some generalizability. The experiments showed that eye movement trajectories have some stability, but are affected by decentrality and randomness. These features are not directly associated with the cultural background of the participant, and also show some novelty compared with previous experimental results. In particular, from the viewpoint of visual information processing, fixational eye movements are a companion process to the visual information processing system, the role of which is equivalent to a sub-switch in a sub-channel of the visual pathway. Further, eye-blinks can play a role similar to frame switching in the whole visual pathway, and the two together can reset visual information. Thus, we think that these results have important implications for the study of visual perception and cognitive psychology.

When we gaze a visual scene, the fixational eye movement mainly refers to eye movement trajectory formed by eyes quickly scanning the scene, also called saccade. In visual perception and visual information processing, however, the exact role of fixational eye movements is still not clear, the view points of psychologists, cognitive scientists and visual computing scientists are not the same, and therefore further research is needed. In human fixational eye movement experiment, the test image is very important. When the test image is flipped in the horizontal direction, participant's eye movement trajectory is also flipped horizontally? To our knowledge, this issue has not been studied in depth. In this paper, we use four different types of test images, such as famous face image, optical illusion image, streetscape and landscape image with vanishing points, and study the relationship between the type of image and human eye movement trajectory, as well as the distribution of fixation points when viewing different test image, especially 
random dot pattern. In experiments we found some interesting results, which shown that the randomness, decentrality and asymmetry are essential feature of human eye movement trajectory.

About what is the role of microsaccade or fixational eye movement, in 1980 to 2000, neurobiologists and psychologists, have had some different views [1,2], for example, Kowler and Steinman think that fixational eye movements are useless, and their views have had great impact over the past 20 years since the publication of their papers $[3,4,5]$ in 1980, after which the study of eye movements was in a stagnant situation. Thereafter, studies by Martinez-Conde, Macknik and Hubel published in 2002 showed that fixational eye movements are useful for visual perception $[6,7,8]$. They tracked eye movements and recorded from visual cortex V1 cells as macaque monkeys fixated, and found increases of cell's activity after microsaccades. So, these authors believe that the vast majority of human visual perceptions are generated via micro-saccades. Moreover, the direction of such subtle movements is not random, but is directed to the area on which participants focus their attention.

In contrast, our research and experiments show that eye movement is an accompanying physiological process of visual channel information processing. The information provided by eye movement trajectories is limited, primary.

During this period, a small amount of papers about fixational eye movement experiments $[9,10]$ have also been published in neuroscience and cognitive psychology. Eye movement trajectory and its experimental techniques have been applied in studies on attention mechanisms and the detection of saliency maps in images [11]. In addition to studying the neural mechanisms of eye movements themselves, such as what are the role of eye movements in the spatial constancy of vision and reducing correlation among visual signals on the retina [12], there are some studies on eye movements suggesting that people have different cognitive processing methods because of different cultural backgrounds [13,14]. Westerners, especially North Americans, more often prefer to analyze objects than Asians. In other words, North Americans are more concerned with target objects than Asians, and tend towards analyzing object properties and then classifying them. In contrast, Asians are considered to have a more holistic view, are more concerned about contextual information and are more willing to make judgments according to object interrelation and similarities than Westerners. There is also some research of great practical value, for example, using experimental data based on tracking eye movements, scientists and engineers have developed fixational eye movement-machine interface software for stroke and hemiplegia patients; after accurately predicting the eye movements of patients using Bayesian inference, software can send action instructions to a helper robot $[15,16]$. There are also many comprehensive studies that combine $\mathrm{AMRI}, \mathrm{EEG}$, and eye tracking to make some diagnosing for mental and mental health patients, to study the development of children's intelligence through the eye-movement trajectory of reading $[17,18]$, and so on.

We pay special attention to the research results mentioned above, as well as the study of eye movement on parafoveal processing in reading and linguistics $[19,20,21]$. However, in terms of the exact role of eye movements in visual information processing, there are very few published related research results, so there is still a need for further discussion (see the Discussion section). The issue includes the following questions:

1. Whether the direction of movement of rapid eye movements (REMs) is random;

2. What does the scattered distribution of fixation point in a wide range mean?

3. Why are eye movement trajectories in a symmetrical image not symmetrical?

These problems are important scientific problems in eye movement research, because they are closely related to visual information processing and cognitive psychology. This paper is also based on our previous work on visual information processing and computer saliency map (including attention mechanism) [22,23,24,25]. We will do some experimental research on the above problems in order to obtain some useful results.

\subsection{Experimental Content and Methods}

When studying these problems, the type of test image is very important. For this reason, we designed four different fixational eye movement experiments. The difficulty of experiments was not the same; in Experiment 1, the test images are the famous portrait of the Mona Lisa [26] (to show a Western women's facial features) and a portrait of a Chinese actress (to show the facial features of Asian women), which are very familiar face images for the participants, which means that it is unnecessary for the participants to carefully judge and analyze the image. Also, the familiarity with these images is not associated with the level of education of the participants, and therefore the eye movement trajectories can well reflect rapid and spontaneous scans of the face image, and which are less affected by the visual pathway of information processing. In Experiment 2, the test image is a visual illusion image, which is important in cognitive psychology research. There are many visual illusion images, such as geometric types of illusions, light and shade types of illusions, movement and contour visual illusions [27,28,29]. Here we mainly focus on contour visual illusions to explore the features of eye movement trajectories when gazing at the bimodal image (that is to say, because gaze direction and fixation point is different, one state of image perceived by participant can be turned into another state, such as the old woman and girl's dual mode) and making a judgment $[24,30]$. Then, in this case, we based the analysis on the relationship between the fixation point and the bimodal optical illusion image. The first important task of the human visual system is to quickly distinguish (within about $100 \mathrm{~ms}$ ) the classification of the visual objects using the contour [24,31] and then further determine its category according to detailed features. Experiments 1 and 2 have one thing in common, which is that both of them use face images; it is generally believed that the eyes in facial images are a naturally salient region. This area is also the main area to which saccades are made. In this experiment, the Asian female eyes (mostly right eye) are indeed a significant region, however, the eye movement test results show that in the Mona Lisa portrait, the ocular region is not a significant area. The number of fixational eye movements to the ocular region is almost zero, and more 
participants' fixational eye movements focus on the mouth region, possibly because the painting is well known for its "mysterious smile".

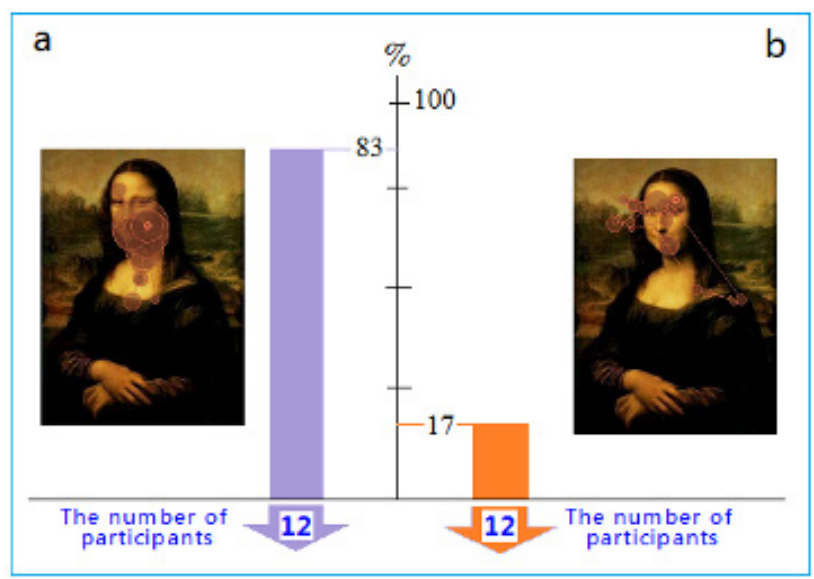

(Mona Lisa portrait is taken from http://en.wikipedia.org/ wiki/MonaLisa)

(a), the proportion including the mouth as a salient area for eye movement trajectory is $83 \%$ of the total experiment; If set $\alpha=0.05$ and the confidence level of $p$ is $95 \%$, it can be find the confidence interval is $(0.54 \sim 0.90)$. (b), the proportion including the eye as a salient area for eye movement trajectory is $17 \%$ of the total experiment. If set $\alpha=0.05$ and the confidence level of $p$ is $95 \%$, it can be find the confidence interval is $(0.049 \sim 0.43)$. The differently-sized brown circles represent the duration time of an eye movement fixation, and a larger circle represents a longer duration time of fixation, and the actual size of each point of light in the picture is the same. When the confidence level of the confidence interval is $\alpha=0.05$. Combined the two confidence intervals in Figure 1 (a) and Figure 1 (b), it can be see that $p_{1}(\mathrm{a})+p_{2}(\mathrm{~b})$ is $0.54+0.43=0.97 \approx 1$; Similarly, $p_{1}(\mathrm{~b})+p_{2}(\mathrm{a})=0.90+0.049 \approx 1$, therefore, the calculation about confidence interval is reasonable.

Figure 1. Experimental results of eye movement trajectory for Mona Lisa portrait [26]

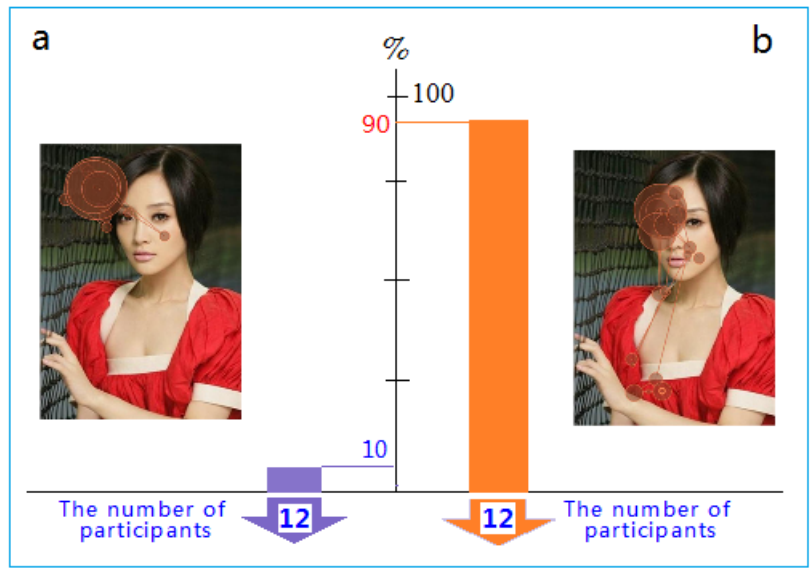

(In Figure 2, the original photo of Li Xiaolu with permission from Li Xiaolu, and the portrait is quoted from http://i04.pictn.sogoucdn.com/8ba6f8ffb9b61 faf)

(a), the eye movement trajectory in which there is no saliency region is $10 \%$ of the total experiment; (b), the eye movement trajectory including the left eye as a salient area is $90 \%$ of the total experiment. Calculate according to the experimental data, in the figure 2 (a) the confidence interval is: $p_{1}(\mathrm{a})=0.02, \quad p_{1}(\mathrm{~b})=0.62,(0.02 \sim 0.38)$. Figure 2 (b) a confidence interval is: $p_{2}(\mathrm{a})=0.38, p_{2}(\mathrm{~b})=0.93 ; \quad(0.62 \sim 0.94)$.

Figure 2. Experiment results of eye movement trajectory for Chinese actress portrait [31]
We use a histogram to represent the percentage including and excluding the eye area in the fixational eye movement experiments, and also provide confidence interval of significance test, as shown in Figure 1, Figure 2, and so on. In contrast, in Figure 2, the experimental results of eye movement trajectories for Chinese female stars [31] show that the proportion of eye movement trajectories in which the right eye portion is salient reaches $90 \%$ over the total experiment, whereas the proportion of eye movement trajectories that does not enter any salient region is $10 \%$. This may be able to be excluded as an invalid test, as the actress's left eye was not looked at by all the participants. However, her right eye was scanned by all participants. Similarly, the Mona Lisa's mouth was scanned with a proportion as high as $83 \%$, with the results reflecting certain stabilities of saccadic eye movement about some features.

In Experiment 2, we use two classic visual illusion test images, the old woman and girl bimodal test picture, and the duck-rabbit bimodal test picture. Participants looked at the two types of illusion image, and also looked at symmetric images, which are horizontal flips of the two illusion images.

In Experiment 3, we used street view images, there were no prominent features in these images. Participants were often able to see the scenes in their daily lives, they were accustomed to do so and naturally did not need much judgment.

Experiment 4 used a landscape image with a vanishing point, from the viewpoint of projective geometry and affine geometry. In such images, the vanishing point is naturally at a significant high point, which is the infinity point used to form stereoscopic perception [22]. This point is highly visible and unique, so it should be a salient area of the image. However, the experimental results conflict with this hypothesis, which raises a problem worth discussing, namely: what is the relationship between the saliency map and eye movement trajectory?

In the experiment, participants also viewed flipped horizontal images, recording their eye movement trajectory, to study the characteristics of eye movement trajectories of a symmetrical image. If the function $f(x, y)$ denotes the test image in the eye movement experiment, then the image after the horizontal flip is just $-f(x, y)=f(-x, y)$, which means that is symmetrical about the $y$-axis in the Cartesian coordinate system. Therefore, after the horizontal flip of the test image, it is flipped horizontal about the $x$-axis in the reference coordinate system, but the relative coordinate position of each part in the test image is not changed. For this reason, flipping the image horizontal will not add new information, and will also not change the original features in the image. In this case, can we make a theoretical judgment in advance about the eye movement trajectory after a horizontal flip?

We will discuss in detail the eye movement trajectories measured in Experiments 2-4 and their relationship to the processing of visual information in the Results section.

\subsection{Significance Test Question}

If the fixational eye-movement experiment is assumed to obey a certain distribution (e.g. $t, u, \chi^{2}, F$ ) in advance, 
or one of its statistical parameters (such as mean $\mu_{0}$ and variance $\sigma^{2}$ ) can be determined, can through the significance test to judge to the design of experiment, to the reliability of the data analysis and experimental results. However, for the eye-movement experiment, there is a great deal of randomness, deconcentration (or scatter) and asymmetry between the eye-movement trajectories of each participant, so that the statistical parameters cannot be determined. Therefore, it cannot be reduced to a known statistical distribution. Figure 3 is the eye movement trajectory in the experiment, it can be seen that the distribution of subject's the fixation point on the image is very scattered. Using the significance test method in the neurobiology, such as fMRI, which uses the normal distribution as the main calculation model, but it is difficult to realize and cannot reflect the distribution of fixation point and the image features about the eye-movement experiments. In order to solve this difficult problem, We according to the main purpose of this experiment is to study whether the features in the test image are the main fixation points of subjects when eyes are in saccade or movement, that is, whether the measured eye gaze at the certain some features of test picture.
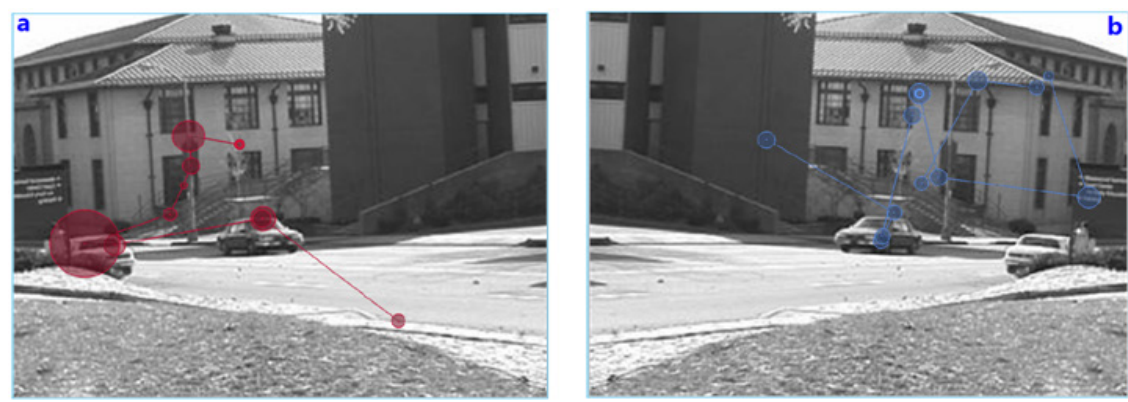

(Figure 3 with permission from the photographer Li Shuzhong \& Song Guangyu)

Figure 3. Distribution of fixation points in an eye movement trajectory of a subject ((a) original picture; (b) picture after horizontally inverted)

Therefore, the "interval estimation" of the $(0,1)$ distribution is appropriate for the processing of the experimental results, as shown in the following equation $[32,33]$

$$
f(x ; p)=p^{x}(1-p)^{1-x}, x=0,1
$$

Where $p$ is the unknown parameter, that is, the probability that some feature in the test image is gazed. The significance test is just the confidence interval $\left(p_{1}, p_{2}\right)$ of $100(1-\alpha) \%$ determined under the selected significance level $\alpha$. The calculation of $p_{1}$ and $p_{2}$ can be found in Ref. $[32,33]$. It should be noted that the significance level $\alpha$ represents the small probability event in the experiment, the appropriate choice of value $\alpha$, can effectively balance the "abandon true" and " choose false" error, in other words, the first type and second type of statistical error. When the number of experimental samples is small, the calculation results will have some errors.

In the four experiments in this paper, the corresponding calculation data are: $\alpha=0.05,(1-\alpha)=0.95, \alpha / 2=0.025$, $z_{\alpha / 2}=1.96, z_{\alpha / 2}^{2}=3.84$, the number of subjects is $n$, the number of subjects in the experiment to gaze at a feature is $m$, the parameter of $(0,1)$ distribution is $\bar{x}^{2}=m / n, p_{1}$ and $p_{2}$ of interval estimation can be obtained by the following formula

$$
\left\{\begin{aligned}
p_{1} & =\frac{1}{2 a}\left(-\mathrm{b}-\sqrt{b^{2}-4 a c}\right) \\
p_{2} & =\frac{1}{2 a}\left(-\mathrm{b}+\sqrt{b^{2}-4 a c}\right) \\
a & =n+z_{\alpha / 2}^{2} \\
b & =-\left(2 n \bar{x}+z_{\alpha / 2}^{2}\right) \\
c & =n \bar{x}^{2}
\end{aligned}\right.
$$


only need a serial number of subjects (thus ensuring no disclosure of privacy), results are only used for scientific research.

\subsection{Participants}

\subsubsection{Experimental Group 1}

In view of the idea that students from the arts and sciences have different logical thinking patterns to each other, to ensure that the experimental results had broad representation, 12 arts and 12 science school graduate students at Beijing Jiaotong University were selected randomly (six male and six female from each) for a total of 24 people The participants were aged 20-24 years (mean age 22 years) with normal vision or corrected to normal vision, and had not previously seen the test picture. Among them, for the above comparison experiment, to avoid the influence of the professional disciplines, the experimental group was divided into two (within-group to ensure an equal number of arts and science students).

\subsubsection{Experimental Group 2}

Five European and American undergraduate and graduate students (two male and three female), and seventeen Chinese undergraduate and graduate students as participants (six men and six women, including one case of data failure), conducted the study in the rapid eye movement laboratory at Beijing Jiaotong University. The average ages of Western and Chinese students were 24.3 years and 25.4 years, respectively. All Chinese participants were born in China, and had completed their undergraduate degrees. The ages and graduate research fields of the participants from the two cultures was matched. Participants were drawn from engineering, life sciences, and business administration graduates.

For this study, the sample size is important. Generally, subjects should not less than 30 people [32,33]. We hope that the number of sample should be a bit more, making the confidence interval of the calculation more accurate, however, there are many specific difficulties, for example, American and European students are very little in our computer college, therefore collected a sufficient number of participants of the voluntary, such as 30, was very difficult. In order to avoid inappropriate results, we do not particularly make comparative analysis between the experimental data of European/American students and Chinese students, because of the less number of American/European students.

\subsection{Test Images}

The four groups of pictures (the famous face images, outline of optical illusion images, ordinary street-view images and landscape images with the vanishing point) are used in the research phase. In the experiment we mainly chose gaze time, number of fixations and eye movement trajectory as parameters, thus providing experimental evidence for the relationship between the types of images and fixation points, and studying and analyzing the relationship between the eye movement trajectories across the image and the same image flipped horizontal.

\subsection{Equipment}

The laboratory instruments used in the experiments included the iView X / RED 250 Type Desktop telemetry eye tracker (SMI, Germany), including an iView PC (test computer), Stimulus PC (image display computer), two infrared light sources and a camera. An automatic infrared tracking system recorded data of monocular motion, with sampling rates of $60 \mathrm{~Hz}, 120 \mathrm{~Hz}$ or $250 \mathrm{~Hz}$. This experimental instrument can automatically record participants' eye movement data, including vertical and horizontal coordinates for each fixation point, start and end time, gaze duration, and pupil diameter or area. It also includes the starting vertical and horizontal coordinate of every saccade, start and end time, duration, saccade distance, and saccade velocity.

\subsection{Experimental Procedure}

(1) Before the experiment began, the operator connected all test equipment required to ensure normal operation of the computer and the eye tracker screen, and calibrated the entire instrument. An experimenter led participants into the eye movement test laboratory, introduced them to the experimental objectives and requirements, and made them familiar with the basic laboratory environment.

(2) The participants sat in a chair $50.0 \mathrm{~cm}$ from the display monitor, relaxed, with their eyes looking straight ahead at the screen. The size of the display was 37.4 inches. Following the directions of the IView software, the experimenter adjusted participant so that they were in an appropriate and comfortable state.

(3) The Participants were told to keep the sitting posture and the head still during the whole experiment. The eyes to look at the computer screen, in this experiment, the participants will see some graphics and answer the questions of the experimental instructors after seeing the graphics.

(4) The experimenter started the Experiment Center program and began the formal experiment, artificially introducing the test pictures, and setting the time of the image playback.

(5) For scaling and calibration, the gaze point of participants was calibrated by 5-point calibration. During calibration, participants were asked to individually look at five red dots randomly presented on a computer screen. Then, whether to start the experiment was selected by the participant.

(6) During the test itself, the experimenter showed the pictures and asked the relevant question, the participants viewed the pictures and answered, and were then shown the next picture. Under the same project directory the same set of experiments were carried out in turn and later the data were uniformly extracted from them.

To eliminate induction effects in participants during questioning (i.e., preconceptions) based on what was asked by the experimenter, counterbalanced questions 
were used. For each set of experiments (for example, in the case of the bimodal test for the old woman and the girl), half of the participants were asked the question, "Do you see an old woman or a girl?", and the other half were asked the question, "Do you see a girl or an old woman?" Such questions with different word orders were used for each set of experimental results.

\subsection{Analysis of the Experimental Results}

The experiments included four groups of different images. One participant's eye-tracking data was poor, and was thus excluded. There were five American and European graduate student participants and 20 Chinese participants in the eye movement experiments, and their experimental eye movement data were analyzed. The data (such as fixation times, fixation duration, saccade counts and regression time) were provided and analyzed using BeGaze software offered by SMI, and were also used to depict static eye movement images and dynamic trajectories. We used this software to generate all kinds of saliency maps based on participants' primary gaze area and the degree of attention paid to it. Based on the exact experimental data, we produced a quantitative analysis about the gaze region. The information obtained in this experiment consisted of:

1. Scan path: point-by-point continuous display of the fixation location, fixation duration and other information for each eye movement. In other words, scan path consists of a series of spatial distributions of fixations and saccades. In images of eye movement trajectories, the circular dot markers show the sequence of fixation points, and line segments show saccades.

2. Screen position of gaze information (gridded areas of interest, it is key areas of interest): display the fixation location information. The fixation region is divided into several squares area, and its color represents gaze duration.

The eye tracking system used in this study can calculate the mean and standard deviation of the statistics of participants' eye movements and related activity data.

How to compare the degree of similarity of eye movement trajectories is a key issue. For each group of pictures, we can choose the area scanned the most times by the participants' eyes as reference points of saliency to calculate the relative distance between each fixation point and the reference point that is the mean deviation from the reference point as an objective standard for comparison.

\section{Results}

In the test experiment containing the bimodal picture of the old woman and the girl, Figure 4 (a) shows the original image [28]. Part A is a salient part of test image, and determines which modal image participants will be seen: the girl's ear or the woman's eyes. Part B is also an important part, and determines whether the participants will see the girl's necklace or the woman's mouth. However, different from part A, it highlights only the old woman's mouth features, it is difficult to be seen as the girl's neck and necklace, and does not have the obvious characteristics of dual-mode conversion.

Therefore, even though half of the participants were asked "Do you see an old woman or a girl?", and the other half were asked "Did you see a girl or an old woman?", this word order did not affect the participants' eye movement trajectory. Most of the participants watched part A, with a proportion as high as $80 \%$. The eye movement trajectory was tested using a symmetric test image as shown in Figures 4 (b) and 4 (c). As the image was flipped horizontally, new eye movement trajectories appear as shown in Figures 4 (c) and 4 (b), which are not exactly the same. In Figure 4 (b) the lower part of the three-point track is more prominent than the one in Figure 4 (c). However, on the whole, the eye movement trajectories in Figure 4 (c) and Figure 4 (b) are very similar. An important result obtained from this set of experiments is: in the case of symmetrical test image, the eye movement trajectory, even if it is not completely symmetrical, is fairly similar (the proportion is $80 \%$ ). When viewing Figure 4 (a), only one case of eye movement did not contain any major features; When viewing Figure 4 (b), the eye movement trajectory contains the same features (girl's ear) to reach 100\% (as shown in Figure 4(d),4(e),4(f) and 4(g)).

In the experiments using the bimodal image "rabbits and ducks"[34,35], flipping the image horizontal or vertical showed very similar eye movement trajectories in the test image after the flip compared with no flip. The trajectory is composed of fixation points around the eye and the front and rear regions of the duck's mouth. Regardless of whether the image is flipped horizontally or vertically, the proportion of such an eye movement trajectory including these three features point is as high as $100 \%$. This result is in fact expected, because when humans look at this bimodal image, the first task is to identify the category of species, and the eyes and mouth naturally become the salient features. No matter which image is perceived by a participant, there is no substantial change the eye movement trajectory as it contains the same significant area. This result further indicates that although a saccade is a rapid scanning process, the eye movement characteristics of the saccade containing the same image feature are stable, and there are no differences caused by the different cultural backgrounds of the EastWest participants.

In Experiment 3, using street view imagery, this class of images has no generally highlighted features. Participants see these scenes often in everyday life and are not required to make too many judgments. Therefore the eye movement trajectory has larger discreteness, the image range scanned is relatively large, the participants' eye movement trajectories are very different, and the fixation points are distributed in most areas of the test image. There are also no rules to follow, and describing such differences using the cross-correlation function gives a cross-correlation coefficient of no more than $10 \%$.

Figure 5 shows a landscape image with a vanishing point. From the view point of projective geometry and affine geometry, the vanishing point of this class images is of high saliency. It is also the infinity point used to form 
stereoscopic perception [22], and is the main region scanned by the eye movement trajectory.

According to the previous analysis, it can be expected that the vanishing point is an important feature point for a saccade. However, the results shown in Figure 5 (c) and Figure 5 (d) are not consistent with the analysis made by means of a saliency map based on attention mechanisms [22]. The proportion of the eye movement trajectory that does not contain the vanishing point is about $25 \%$, as shown in Figure 5 (e). The proportion of fixation vanishing point and its time is shown in Figure 5 (f).
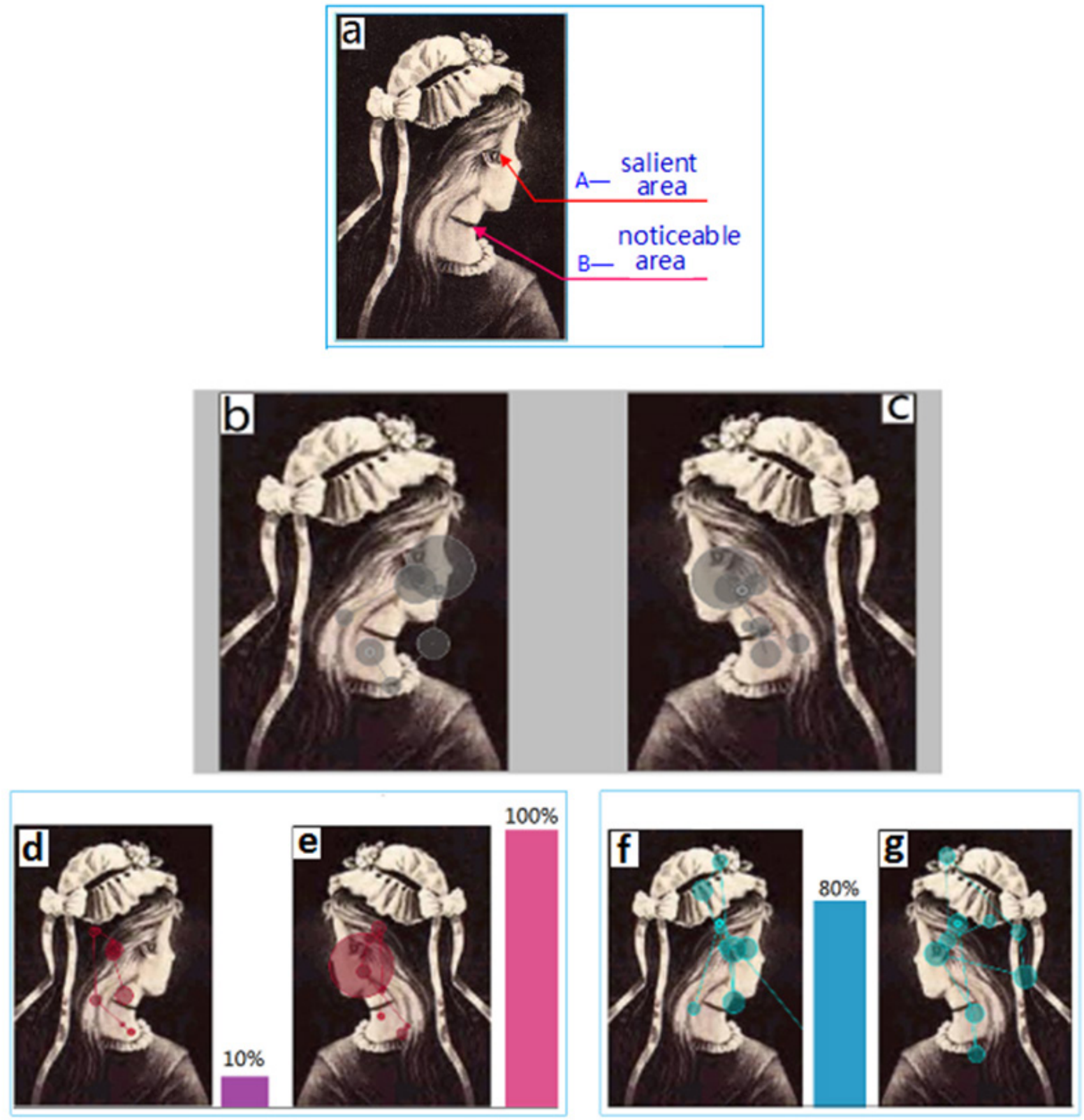

(The portrait is quoted from Kornmeier, J. \& Bach, M. Object perception: When our brain is impressed but we do not notice it. Journal of Vision. 9(1):7, $1-10,2009)$.

(a) The key feature point in the bimodal image, (b) eye movement trajectories for the bimodal image, (c) flipped horizontal image and eye movement trajectories after Figure 4(b) is flipped horizontally; (d) eye movement trajectory only including B-point of key feature (the old woman's mouth, see,4(a); the number of subjects whose fixation point in the trajectory of the eye contains only the features of the old woman's mouth, when $\alpha=0.05$, the confidence interval of (a) is:(0.02 0.38). (e): after the horizontal reversal of figure (a), eye movement trajectory including A-point and B-point of key feature ( the eye region and mouth region of the old woman); the number of subjects whose the fixation point of the eye-movement trajectory contains the eye region and mouth region of the old woman (all the participants watched the old woman's eyes); (f) and (g): the symmetric image of the eye movement trajectory, when $\alpha=0.05$, interval of the confidence interval is: $(0.48 \sim 0.89)$.

Figure 4. Similarity analyses of eye movement trajectories for bilateral symmetrical picture and the bimodal experiment of the old woman and the girl, the ratio of the different fixation points in the eye-movement trajectory (with the old woman as the modal) [28] 


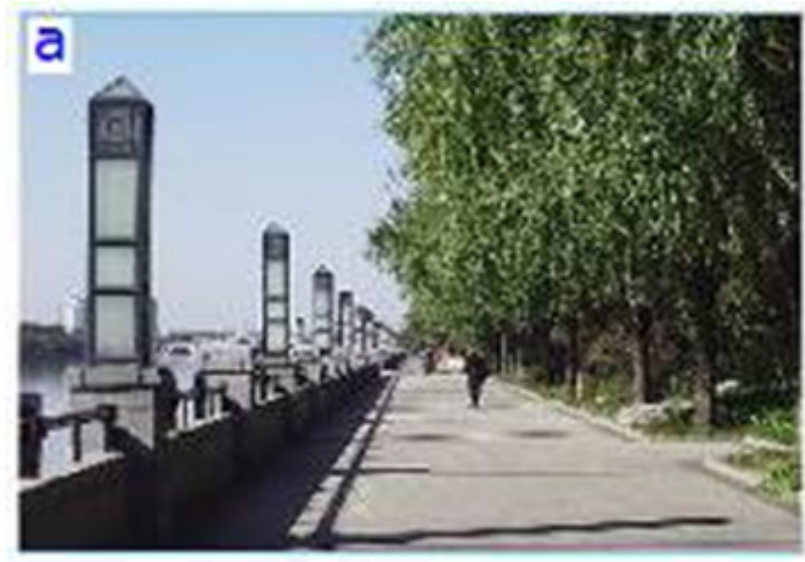

(a) actual landscape images

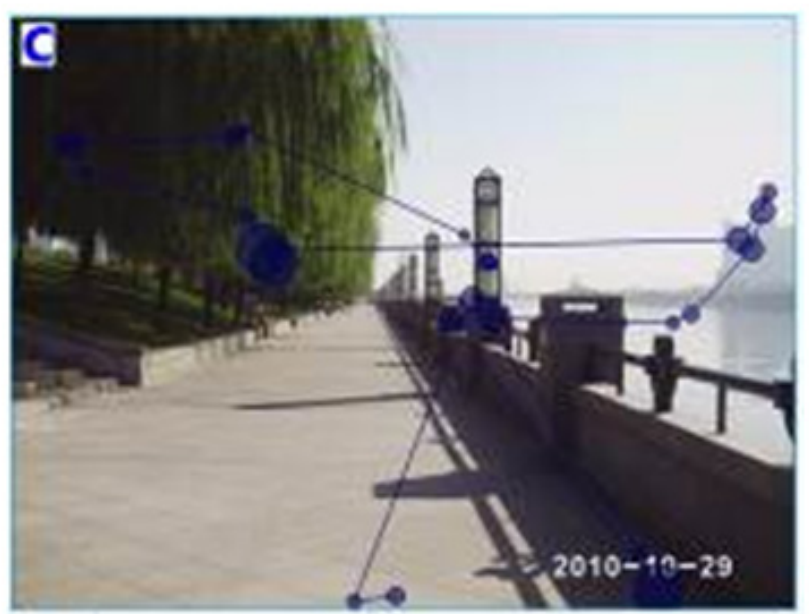

(c) eye movement trajectory (does not contain vanishing point)

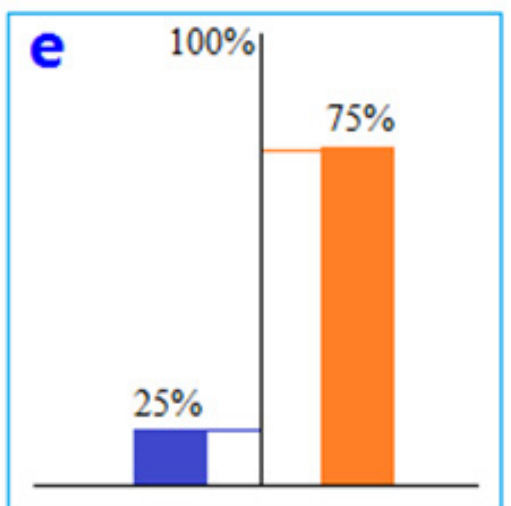

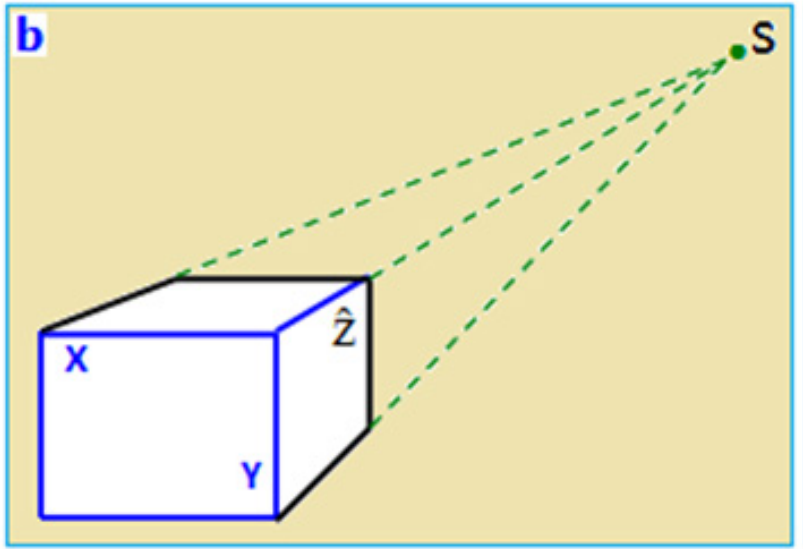

(b) schematic diagram of the vanishing point

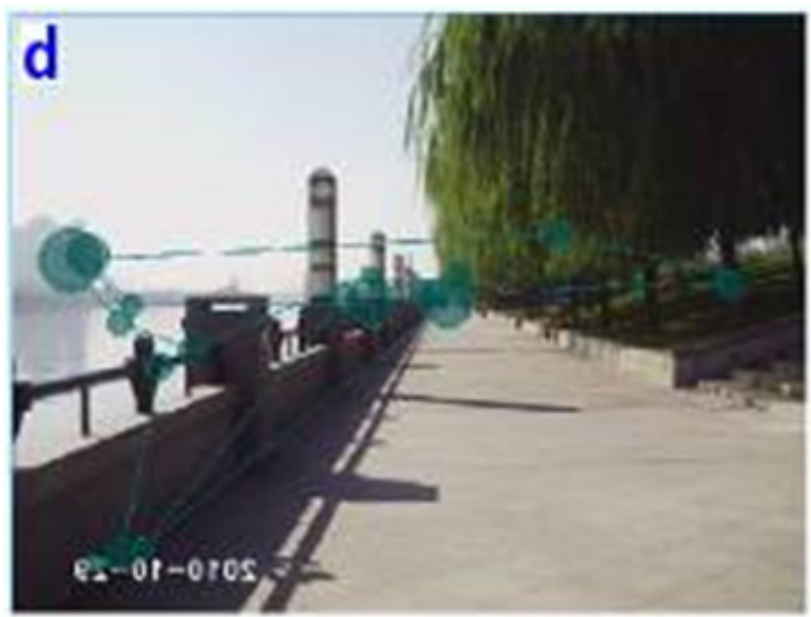

(d) eye movement trajectory of symmetrical image with (c) (which contains the vanishing point)

(e) eye movement trajectory does not contain the vanishing point (blue), the ratio of the participant is $25 \%$, the ratio of the participant including the vanishing point is $75 \%$ (orange), when $\alpha=0.05$, the significance test is: with respect to blue case, $p_{1}=0.08, p_{2}=0.50$, interval estimation is $(0.08 \sim 0.50$ ); relative to orange case is $p_{1}=0.45, p_{2}=0.86$, interval estimation is $(0.45 \sim 0.86)$. ( $\mathrm{f}$ ) proportion and duration time of fixation points of subjects to look at the vanishing point. To note that, since the sample size is not large, thus, interval estimation is relatively wide, which have been discussed in detail in the text.

(The original photos in Figure 5 (a), (c) and (d) with permission from the photographers Li Shuzhong \& Song Guangyu)

Figure 5. The landscape image with vanishing point and its eye movement trajectory

\section{Discussion}

We know that human eye movements fall into three basic types: fixation, saccades and pursuit movement.
When reading or looking at a picture, to clearly see an object, both eyes must maintain a certain position, thus making an image of the object on the retina. This is called fixation, and in this case, eye gaze can remain relatively 
stable. The function of saccades is to change the fixation point, so that the object fixated on is in the most sensitive area of the retina, i.e., near the center of the fovea, so that you can more clearly see the content you want to see. Usually we do not feel our eyes making saccades, and the feeling we have is one of smooth movement. In fact, our eyes are always fixating on part of an object for some time. They then jump to another part, and then look for new targets; pursuit movement refers to this situation $[36,37]$. We know that information processing in the brain is parallel $[38,39]$. When gazing at an object, the brain is also simultaneously performing other tasks. The randomness of eye movement trajectories and the attention to features in images produce stability of scanning. Further, when looking at garden scene (Figure 5), different viewing habits of participants form a decentralized distribution or scattered features of fixation points (that is to say, when saccade, the fixation point stays in many areas of the image being watched, the scanned image area is large, the fixation points are scattered in different places). To produce definite conclusions on this issue, further study and tests are necessary, perhaps on the eye movement trajectories of anesthetized patients or those with dementia.

It is generally believed that the human visual system has two information processing approaches: bottom up and top-down. The bottom-up process receives light intensity array signals of natural scenes and processes information received, which is usually called data-driven processing (this is equivalent to the low-level salience of the visual stimulus, such as luminance, contrast, and color [39-43]). The top-down process is feedback information processing by the perception of an object via knowledge and experience, also known as task-driven processing (this is equivalent to high-level task demands and prior knowledge [43]). Because the amount of knowledge and experience of percepts is different among participants, it will impact their eye movement trajectory. Our experiment shows that rapid eye movement is only a physiological process associated with visual information processing, so information provided by eye movement trajectory is very limited and preliminary.

In the experimental test of the Mona Lisa portrait's symmetrical image, the decentrality and asymmetry of the eye movement trajectories (i.e., the fixation point does not shift to the corresponding new position as the image is flipped) indicate that the brain is processing the information in parallel, that is, the knowledge and experience of the participant about an object is used as top-down feedback, which directly affects the process of visual attention. Different knowledge and experience among participants will affect their attention to objects. Eye movements are accompanying physiological responses associated with attention mechanisms. Within the main direction and the range determined by the participant's attention, eye movements (saccades) were seemingly random scanning. We did not find eye movement trajectories that were identical or similar to each other in these tests. At different times, multiple tests on the same participant on the same image showed eye trajectories that were not the same or not substantially the same, which is good evidence showing that eye movement trajectories for different groups, as well as for the same participants at different test times, are all different and random. In fact, we can use the random dot pattern as a test image, as this diagram does not have any image features, participant's fixational points in eye movement trajectory is capable of displaying the intrinsic random and scattered distribution, as shown in Figure 6.
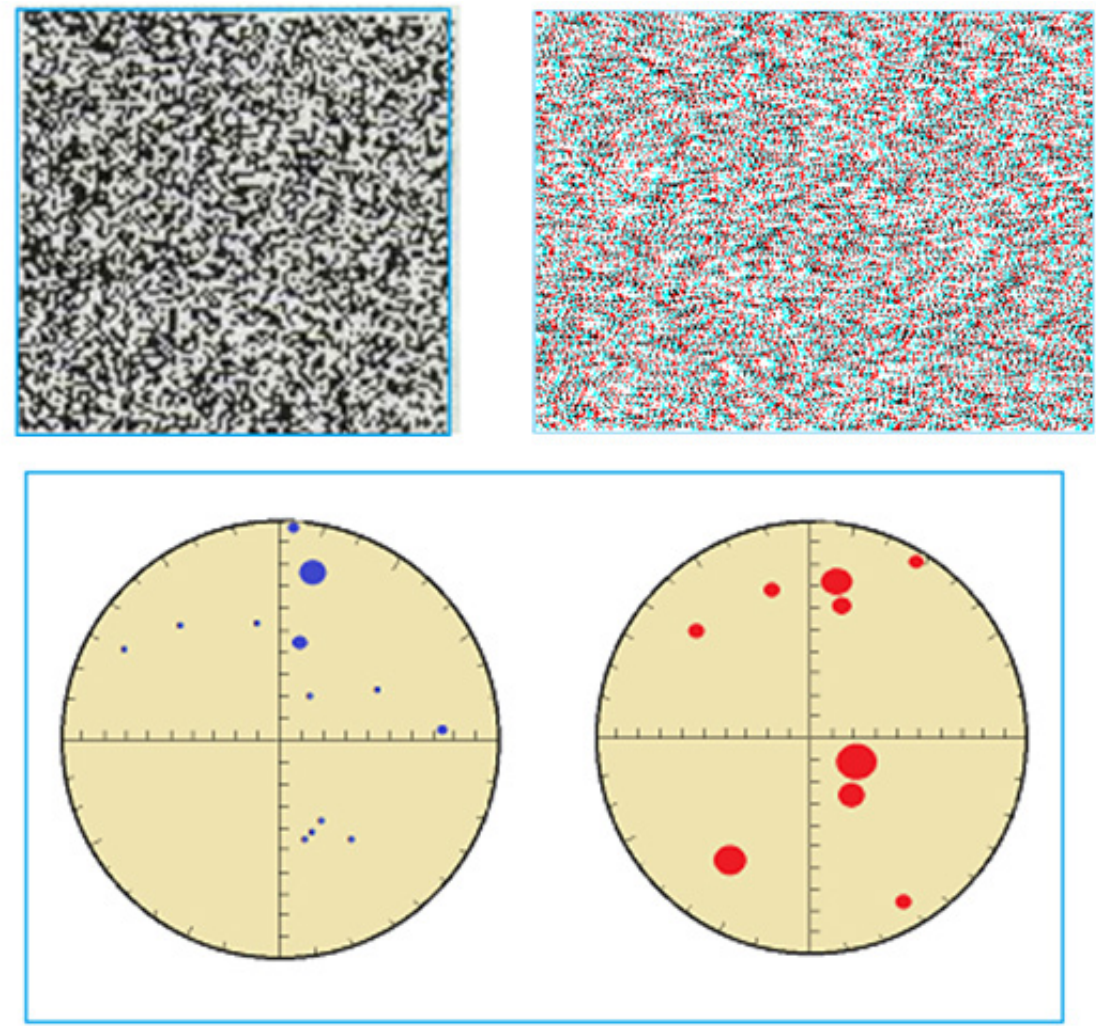

Figure 6. When random dot pattern as a test image, different spatial distribution of fixational points of the eye movement trajectory for any two paticipants, in which the size of the dot represents fixational time length of their stay 


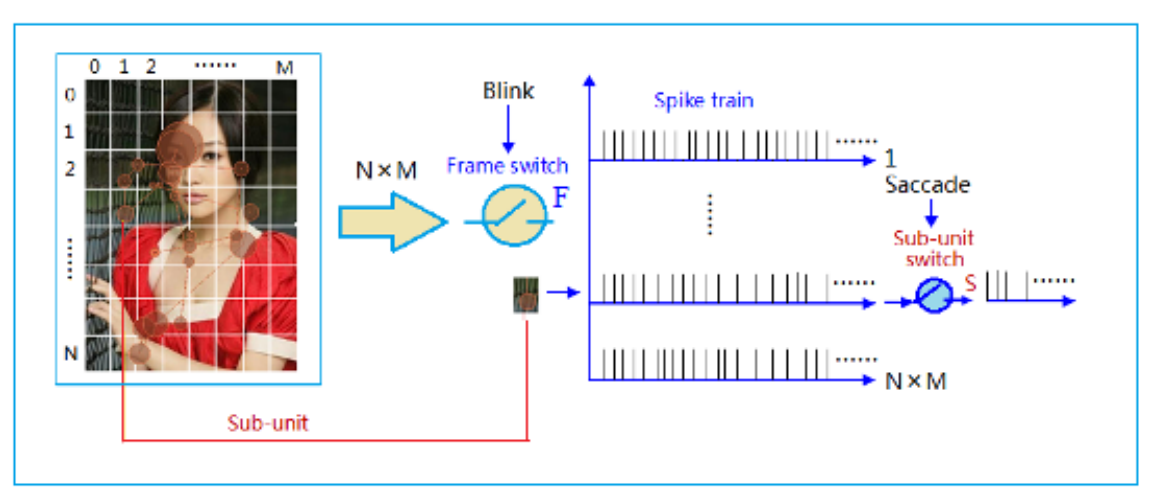

(In Figure 7, the original photo of Li Xiaolu with her permission from Li Xiaolu, and the portrait is quoted from http://i04.pictn.sogoucdn.com/8ba6f8ffb9b61faf)

Figure 7. A schematic diagram of blinking and the role of eye movements in visual information processing $[23,24,48]$

There is a phenomenon associated with eye movements worthy of attention: blinking the eyes [44,45,46,47], together with eye movements, will play a role in resetting or refreshing the transmission of the external light intensity array signal into the visual pathways, immediately restoring the array signal [24,39]. According to the viewpoint of visual information processing $[23,24]$, the whole visual image can be divided into $\mathrm{M} \times \mathrm{N} \approx 10^{6}$ units (or image primitive) by the size of the receptive field of a photoreceptor cell in the retina. The formed light intensity array signal of visual input is further processed by the functional columns in the visual cortex. A blink rate of 15-20 times per minute is equivalent to a frame switch for the entire visual pathway (F), and eye movements can play role as a sub-channel switch (S) in the visual pathway. For those units (primitive) that are not scanned, their resets are achieved via blinks (switch S), corresponding to the schematic shown in Figure 7. Their role is to prevent the neuron's impulse fire train (spike train) from lasting for too long, which is thought to prevent the adaptation of neurons $[6,7,8]$. In fact, external stimuli can excite a neuron's firing for only a short time in the visual cortex [48], and once it is interrupted by blinking and eye movements, it begins to fire a new impulse. As for the stability of perception, existing studies have shown that this is related to the contribution of the thalamus cortex, but this issue is beyond the scope of this paper $[37,49]$.

Eye movement is actually equivalent to the switch of neuron firing spike train, if without the switch, the neurons will continue to fire the spike train pulse, due to consume large amounts of neuron's energy, the frequency of firing spike train is getting lower.

\section{Conclusions}

In four kinds of eye movement experiments, we selected some representative face pictures as test images, and then analyzed data about eye movement trajectories obtained from these tests, and came to the following conclusions:

1. Eye movement trajectories for landscape and street scene images possess features that are random and discrete.

2. The scanned area of the image using eye movements is large, and eye movement trajectories did not point to any particular direction.
3. For the face images, animal (duck-rabbit) bimodal image and their flipped horizontal images, the eye movement trajectory showed symmetry and stability, with the fixation mainly focusing on the main feature points (eyes or mouth).

When gazing at a scene, the eyes steadily fixate on the object in view for some time, and the eye movements adjust the fixation point to the fovea region to produce a clear image. Therefore, rapid eye movement is an accompanying physiological process in the visual information processing channel (or visual pathway); information provided by eye movement itself is only limited and primary. Therefore, the saccade is not only a physiological response, but also a need for visual information processing. We know that the visual system can extract information by means of the lateral inhibition of function and the receptive field of photoreceptor cells (cone and rod cells). The eye movement trajectory reflects the eyes' rapid scanning and discontinuous gaze process, although it also reflects the positioning of attention to a certain extent. However, fixation information is very limited, so under normal viewing conditions, we rarely feel how the eye moves.

In addition, the test image was flipped horizontally, so only the reference axis of the coordinate system was flipped horizontally, and the relative coordinate position of each portion of the image was unchanged. The image flip does not add new information. In this case, flipping the image horizontally means that the entire facial image will also be flipped horizontally, that is, the image features and their locations in the image simultaneously and horizontally flip. So does the eye movement trajectory change? In other words, which is more important for the viewer, the image features or their locations in the image? The results from the symmetrical image experiments of the Mona Lisa portrait and actress showed that when the image was flipped for participants, their eye movement trajectories showed some differences compared with the original image; therefore, it is impossible to answer the question of whether the features of the image or their positions in the image are more important.

In fact, external stimuli cause neurons' spike train firing in the visual cortex V1, which only lasts a certain amount of time, interrupted by blink and eye movements, and then begins a new round of spike train firing under external stimuli [29]. It is worth noting that the experiments in this paper show that the distribution of eye 
fixation points is random, scattered and asymmetric, which is an important result, because it can explain both neuroscience and psychological views. If its distribution is deterministic, centralized, and symmetrical, then in the eye movement it must coincide with the direction of visual gaze (For example, the direction of the visual axis). When the effect of blinking is not taken into account, the effect of micro saccades is only equivalent to superimposing of the micro-switch on visual gaze, only for a fixed image primitive have an impact, at least in one functional column of visual cortex to the neurons to play the role of instantaneous switch, so that it is excitated and in re-active state, thus increasing the spike train firing rate in the certain time interval. Psychologists used to do studies about eye movements according to behavior patterns for some visual tasks (such as aim at targeting in shooting, thread a needle, etc.). But, due to the visual tasks are short-term with mental high concentration, the eye moving and blinking cannot appear. Naturally, it is difficult to draw some effects on the psychological activities or behavior. And neuroscientists can effectively measure the activity state of individual neurons, that is, the change and increase of spike train firing rate. So, it is easy to find the eye movement or micro saccades associated with neurons' active status. Of course, cognitive psychologists have now identified eye movement study is meaningful and have used eye movements for research in areas such as reading and mental development, and many results have been achieved [17,18,19,20,21].

According to our experimental results, we have a clearer understanding on the role of eye movement and blink. From the viewpoint of visual information processing, eye movement and blink were respectively equivalent to the switch $\mathrm{F}$ in visual pathway and the micro-switch $\mathrm{S}$ in a channel, which is known to have about one million parallel channels (see Figure 8 and reference [23] ). The distribution of eye fixation points in eye movement or micro saccades is random, decentralized and asymmetric, which corresponds to the distribution of the switch $\mathrm{S}$ is just distributed. This is consistent with the known biological structure of the visual channel, that is, the combined action of switch $\mathrm{F}$ and micro-switch $\mathrm{S}$ can interpret neuroscience research results and also explain the psychological the observations.

\section{Acknowledgements}

This research is supported by the National Natural Science Foundation of China (Grant No. 41675010, No.61271425 and No. 61473031) and the Beijing Youth Talent Plan (Grant No. YETP0546).

\section{Competing Financial Interests}

The authors declare no conflict of interest.

\section{References}

[1] Munoz, D.P. (2002). Commentary: Saccadic eye movements: Overview of neural circuitry. Prog Brain Res. 140. 89-96.
[2] Sparks, D.L. (2002). The brainstem control of saccadic eye movements. Rev Nat Neurosci. 3, 952-964 .

[3] Kowler, E. \& Steinman, R. M. (1977). The role of small saccades in counting. Vision Res. 17, 141-146.

[4] Kowler, E. \& Steinman, R. M. (1979). Miniature saccades: eye movements that do not count. Vision Res. 19, 105-108.

[5] Kowler, E. \& Steinman, R. M. (1980). Small saccades serve no useful purpose: reply to a letter by R. W. Ditchburn. Vision Res. 20, 273-276.

[6] Conde, S. M., Macknik, S. L. \& Hubel, D. H. (2004). The role of fixational eye movements in visual perception. Nature Reviews Neuroscience. 229-240.

[7] Conde, S. M., Macknik, S.L. \& Hubel, D. H. (2002). The function of bursts of spikes during visual fixation in the awake primate lateral geniculate nucleus and primary visual cortex. PNAS. 99, 13920-13925.

[8] Conde, S. M., Millan, J.O. \& Macknik, S. L. (2013). The impact of micro- saccades on vision: Towards a unified theory of saccadic function. Nature Reviews Neuroscience. 14, 83-96.

[9] Engbert, R., Mergenthaler, K., Sinn, P. \& Pikovsky, A. (2011). An integrated model of fixational eye movements and microsaccades. PNAS. 108, 765-770.

[10] Naoko Inaba. \& Kenji Kawano. (2014). Neurons in cortical area MST remap the memory trace of visual motion across saccadic eye movements. PNAS. 111, 7825-7830.

[11] Schütz, A.C., Trommershäuser, J. \& Gegenfurtner, K.R. (2012). Dynamic integration of information about salience and value for saccadic eye movements. PNAS. 109, 7547-7552.

[12] Segal, I.Y. et al. (2015). Decorrelation of retinal response to natural scenes by fixational eye movements. PNAS. 112, 3110-3115.

[13] Chua, H. F., Boland, J. E. \& Nisbett, R. E. (2005). Cultural variation in eye movements during scene perception. PNAS. 102, 12629-12633.

[14] Goeke, C. et al. (2016). Cultural background shapes spatial reference frame proclivity. Scientific Reports. 5(11426), 1-13.

[15] Graf, A. B. A. \& Andersen, R. A. (2014). Brain-machine interface for eye movements. PNAS. 111, 17630-17635.

[16] Levy, R., Bicknell, K., Slattery, T. \& Rayner, K. (2008). Eye movement evidence that readers maintain and act on uncertainty about past linguistic input. PNAS. 105, 10131-10136.

[17] Hanke, M. et al. (2016). A studyforrest extension, simultaneous fMRI and eye gaze recordings during prolonged natural stimulation. Scientific Report.160092, 1-15.

[18] Namazi, H., Kulish, V. V. \& Akrami, A. (2016). The analysis of the influence of fractal structure of stimuli on fractal dynamics in fixational eye movements and EEG signal. Scientific Reports. 6, 26639.

[19] Schotter, E. R., Lee, M., Reiderman, M. \& Rayner, K. (2015). The effect of contextual constraint on parafoveal processing in reading. Journal of Memory and Language. 118-139.

[20] Watson, F.S., Leekam, S.R., Benson, V., Frank, M.C. \& Findlay, J. (2009). Eye-movements reveal. Neuropsychologia. 47, 248-257.

[21] Satoko Hisanaga., Kaoru Sekiyama.,Tomohiko Igasaki. \& Nobuki Murayama. (2016). Language/ Culture Modulates Brain and Gaze Processes in Audiovisual Speech Perception. Scientific Reports. 6, 35265.

[22] Zhao Songnian. et al. (2014). The representation of visual depth perception based on the plenoptic function in the retina and its neural computation in visual cortex V1. BMC Neuroscience. 15, $1-17$.

[23] Songnian Z., Qi Z., Zhen J., Guozheng Y. \& Li Y. (2010). Neural computation of visual imaging based on Kronecker product in the primary visual cortex. BMC Neuroscience. 11, 1-14.

[24] Songnian Z., Qi Z., Zhen J., GuoZheng Y. \& Li Y. (2010). A computational model of early vision based on synchronized response and inner product operation. Neurocomputing. 73, 3229-3241.

[25] Zhao S., Xiong X., Yao G. \& Fu Z. (2003). A computational model as neurodecoder based on synchronous oscillation in the visual cortex. Neural Computation.15, 2399-2418.

[26] Devore, J.L. (2015). Probability and Statistics for Engineering and the Sciences. (Ninth Edition CENGAGE Learning, Boston, MA, USA, 276-309; 361-408).

[27] Ross, S. M. (2014). Introduction to Probability and Statistics for Engineers and Scientists.(Fifth Edition, Academic Press of Elsevier, MA, USA, 235-285). 
[28] Mona Lisa portrait is taken from http://pic.sogou.com; which is public web in China. Of course, portrait of Mona Lisa can be taken from other web, such as:

www.megamonalisa.com/;www.pbs.org/treasuresoftheworld/mon a lisa/mmain.html; https://en.wikipedia.org/ wiki/Mona_Lisa.

[29] Corney, D.R. \& Lotto, B. (2007). What Are Lightness Illusions and Why Do We See Them? PLoS Computational Biology. 3, 1790-1800.

[30] The old woman and the young girl illusion can be taken from following web: http://mathworld.wolfram.com/YoungGirlOldWomanIllusion.html.

[31] Frisby, J. P. \& Stone, J. V. (2010). Seeing: The Computational Approach to Biological Vision. (Oxford University Press. London: Houghton Mifflin).

[32] Perrinet, L.U. \& Bednar, J. A. (2015). Edge co-occurrences can account for rapid categorization of natural versus animal images. Scientific Reports. 5, 1-7.

[33] Figure 2 with permission from Li Xiaolu ; and the portrait is quoted from http://www.tupianzj.com/mingxing/xiezhen/xuqing/; it also can be taken from other web: http://weibo.com/lixiaolu

[34] Cambers, D. \& Reisberg, D. (1985). Can mental images be ambguous?, J. Exp. Psychol.: Human Perception and Performance. 11, 317-328.

[35] Franz, V.H. \& Scharnowski, F. G. (2005). Illusion effects on grasping are temporally constant not dynamic. J. Exp. Psychol.: Hum Percept Perform. 31, 1359-78.

[36] Munoz, D.P. (2002). Commentary: Saccadic eye movements: Overview of neural circuitry. Prog. Brain. Res. 140,89-96.

[37] Ostendorf, F., Liebermann, D. \& Ploner, C.J. (2010). Human thalamus contributes to perceptual stability across eye movements. PNAS. 107, 1229-1234.

[38] Bichot1, N.P., Rossi, A. F. \& Desimone, R. (2005). Parallel and Serial Neural Mechanisms for Visual Search in Macaque Area V4, Science. 308, 529-534.
[39] Marr, D. (1982). Vision, Computational investigation into Human representation and processing of visual information. (San Francisco: W H Freeman and Company).

[40] Itti, L. (2005). Models of bottom-up attention and saliency In Neurobiology of Attention. (eds Itti,L., Rees, G. \& Tsotsos.) 576582 (Academic Press, Elsevier Inc., San Diego, CA.).

[41] Buschman, T. J. \& Miller, E. K. (2007). Top-down versus bottomup control of attention in the prefrontal and posterior parietal cortices. Science, 315, 1860-1862.

[42] Schall1, J. D., Paré, M. \& Woodman, G. F. (2007). Comment on "Top-Down Versus Bottom-Up Control of Attention in the Prefrontal and Posterior Parietal Cortices". Science. 318, 44.

[43] Zou Qi., Zhao Songnian., Wang Zhe. \& Huang Yaping. (2012). A neural computational model for bottom-up attention with invariant and overcomplete representation. BMC Neuroscience. 13, 1-22.

[44] Tamami Nakano., Makoto Kato., Yusuke Morito., Seishi Itoi. \& Shigeru Kitazawa. (2013). Blink- related momentary activation of the default mode network while viewing videos. PNAS. 110, 2702-706.

[45] Bridgeman, B. \& Palca, J. (1980). The role of microsaccades in high acuity observational tasks. Vision Res. 20, 813-817.

[46] Moore, T., Tolias, A.S. \& Schiller, P.H. (1998). Visual representations during saccadic eye movements. PNAS. 95, 8981-8984.

[47] Moore, T. (1999). Shape Representations and Visual Guidance of Saccadic Eye Movements. Science. 285, 1914-1917.

[48] McFarland, J. M., Bondy, A.G., Cumming, B.G. \& Butts, D. A. (2014). High-resolution eye tracking using V1 neuron activity. Nature Communications 5, 1-12.

[49] Higgins, E. \& Rayner, K. (2015). Transsaccadic processing: stability, integration, and the potential role of remapping. Atten Percept Psychophys. 77, 3-27. 